\title{
Modeling and analysis of hybrid controller by combining MFB with FLC implemented to ultracapacitor-based electric vehicle
}

\author{
RAGHAVAIAH KATURI, SRINIVASARAO GORANTLA \\ Department of Electrical and Electronics Engineering, \\ Vignan's Foundation for Science, Technology, and Research, \\ Vadlamudi, Guntur, Andhra Pradesh, INDIA
}

\begin{abstract}
An optimized energy management strategy is designed for a hybrid energy storage system (HESS) to drive the hybrid electric vehicle (HEV) / electric vehicle (EV). Combination of battery and Ultra-Capacitor (UC) forms the HESS for an HEV / EV which will enhance the acceleration performance of the electric motor, reduced battery charge-discharge cycle and improves the driving range. The main aim of the proposed method is to design a control approach for automatic smooth switching between sources of HESS corresponding to the speed of the motor. To achieve the main objective, a new controller is designed with four math functions and programmed separately corresponding to the speed of the motor termed as MFB controller. The fuzzy logic controller is used to obtain the controlled gate pulses for Uni-directional (UDC) and Bi-directional (BDC) converters, those are placed at the battery and UC end. The MFB and Fuzzy logic controllers work together to attain a smooth transition between the battery and UC related to the speed of the electric motor. Finally, the effectiveness of the control strategy is validated with four modes of operation in MATLAB/Simulink.
\end{abstract}

Keywords: Hybrid electric vehicles(HEVs), battery, ultracapacitor (UC,) Bidirectional Converter (BDC), Unidirectional converter (UDC), Battery, Ultracapacitor, Math Function Based (MFB) Controller, Fuzzy logic Controller (FLC).

[

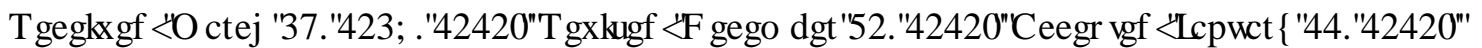

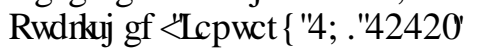

\section{Introduction}

In the transportation system, electric vehicle technology is one of the most feasible ones to protect the atmosphere from the flue gases and to reduce the energy crises. Electric vehicle technology is bagged with some conventional drawbacks, in that driving range of the electric vehicle is the major problem. The conventionally electric vehicle is feed with battery or fuel cell for its successive propulsion, which gives better result during average power requirements and for transient power generation, frequent charging and discharging leads to take more cost for maintaining the battery. To avoid such problems UC has been introduced for high-power requirements, moreover to reduce the burden on the battery during a transient period of the electric vehicle. HESS has been formed with battery and UC for all type of road condition. The major task in this HESS is that is a smooth transition between the battery and UC. For obtaining the smooth transition between energy sources in HESS many controllers have been designed including a conventional controller like PI, PID, etc.

The main issue in HESS based electric vehicle is the energy management between the battery and the UC depending upon the vehicle dynamics. An adaptive fuzzy logic based controller has been designed for proper energy splitting between energy sources in HESS. Fuzzy logic is used to solve the real time control issue after that this does not require driving cycle dynamics [1]. To optimize the operation process of the HESS in the electric vehicles fuzzy logic controller has been proposed. The rule base can be done using Kalman filtering algorithm to know the state of power and state of charge, from this knowledge optimized power value can be found which can be supplied by the battery with that state of health of the battery will improve [2]. During high power density requirement, energy storage is the main issue from electric vehicles for its successful operation. The provided battery must be low cost, having a long life cycle and small in size. But batteries having low power density, and fortunately, UCs are available with high power density.
So batteries lagged power can be provided by the UC during peak load time of the electric vehicle. Energy control can be achieved from DC-DC converters by providing proper pulses to the switches based on the state of charge of battery and UC with the help of a PI controller [3]. Flexible energy management control has been studied for HESS. An inversion mode control scheme has been proposed and it requires the distribution input for its proper energy sharing between batteries an ultra-capacitor. Using different distributed inputs several controllers have been checked without changing the control scheme. Some real-time control schemes also proposed and compared with existed control scheme [4]. FLC based HESS has been designed to reduce the power rating of the BDC by meeting the power requirement of the DC bus. Total four Fuzzy logic controllers have been designed for different modes of operation of electric vehicle which depending upon the speed of the EV. Designed FLC calculates the power requirement between the battery and ultra-capacitor [5]-[6]. A fuzzy logic supervisory wavelet-transform frequency decoupling-based energy management strategy has been proposed to the powertrain to propel the two different power rating machines having HESS with battery and ultra-capacitor. The adopted method guarantees the rated power from the battery and transient power from the ultra-capacitor, power splitting between sources can be done based on the torque requirement [7]-[8]. A new control structure is proposed by considering a variable rate -limit function and implemented to HESS. With proper energy splitting between energy sources of HESS, the major source of life is enhanced. The normal power need of the load is achieved by the major source whereas sudden power will send from the supporting source. [10]. With fuel cell and UC HESS is constructed, to change the energy source related to load requirement a new polynomial based approach is used. UC is used for sudden power supply on the other hand main source will meet the normal power of the load [11]. A controller is designed and implemented of power-sharing of energy source present in the vehicle. The considered HESS mainly consisting of UC and battery, here the UC is used for 
quist starting of the vehicle with that the main source. [12]. This work mainly concentrates on designing the controller for a smooth transition between the battery and UC. The MFB controller is designed and integrated with the fuzzy logic controller to attain the goal of this work. Here the MFB controller and fuzzy works together and generate the switching signals to the converters to regulate the power flow from battery and UC.

\section{Proposed System Model}

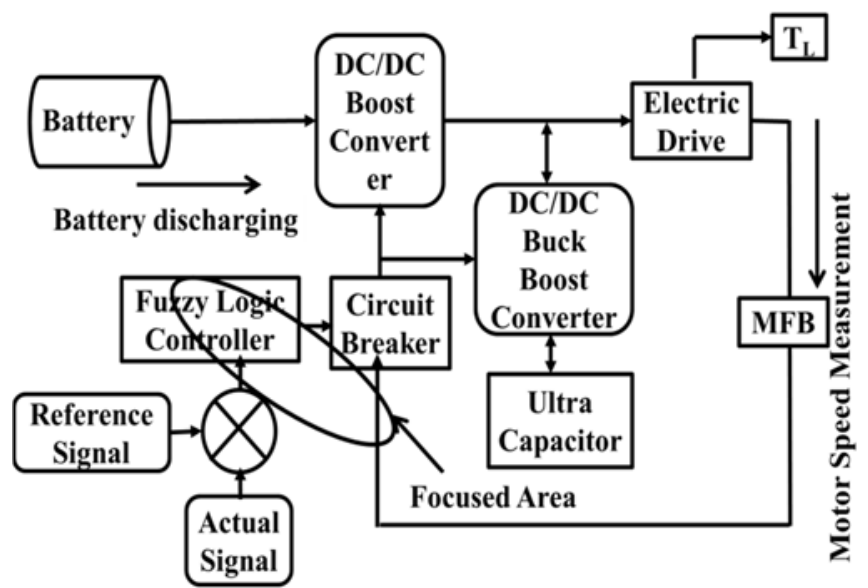

Figure 1. Implemented proposed circuit block diagram representation

The proposed scheme mainly having converters, controllers, two different sources and BLDC motor in the form of load. Here UDC and BDC are connected separately at both the sources battery and UC, end. From that chosen two sources, the battery will able to give the normal power to the load, reversely UC will provide the aggressive power at abnormal conditions, this attempt will enhance the batteries life. With the proposed scheme the demanded switching signals of the converters are produced related to the EVs dynamics.

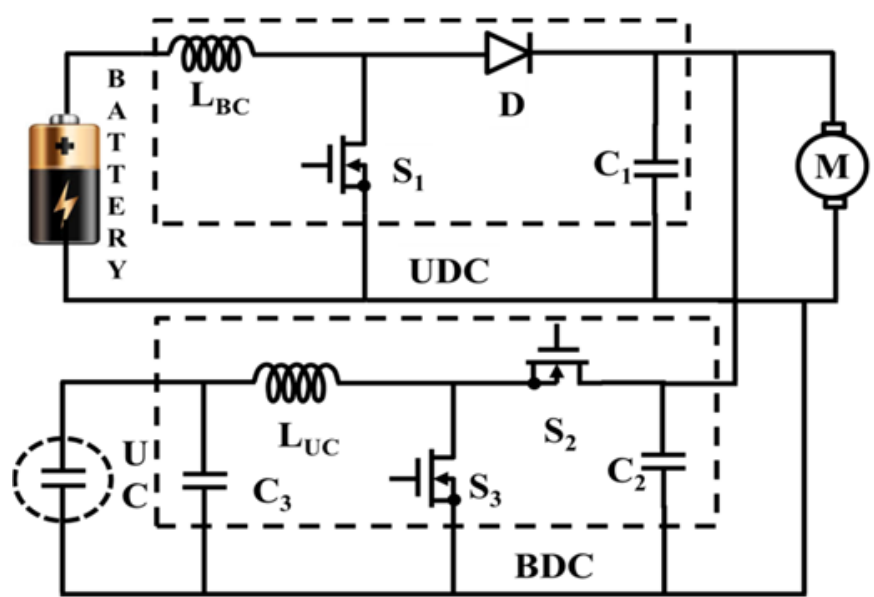

Figure 2. Main circuit with converters and sources

Figure 2 represents the converter model of HESS where Uni-Directional Converter (UDC) and Bi-Directional Converter (BDC) models have been preferred by means of MOSFET switches. Mainly three switches S1, S2, and S3 plays the key role during changing of the sources from two another corresponding to the behavior of the vehicle. Among three switches S2, S3 are present in BDC to perform both buck and boost operation. In the same way, S1 is exclusively present in UDC to perform boost operation. The controlled switching signals production will take place based on the proposed control scheme.

\section{Analysis and Modelling of HESS}

\subsection{Battery model}

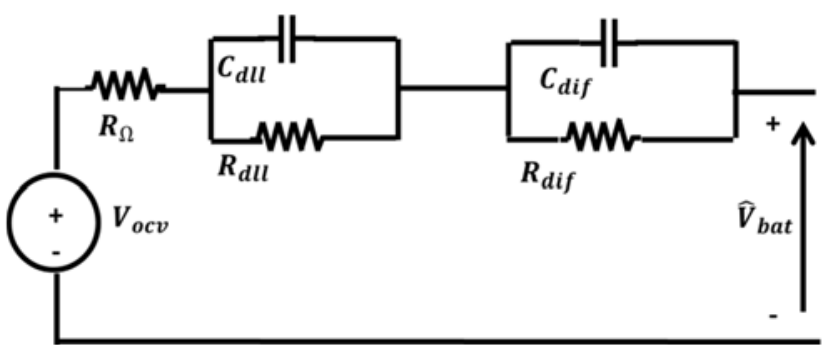

Figure 3. The general structure of the battery

Various parameters of the battery are

$$
\begin{gathered}
\mathrm{f}_{1}\left(\mathrm{~V}_{\mathrm{t}}, \mathrm{i}_{1}, \mathrm{i}\right)=\mathrm{E}_{0}-\frac{K \mathrm{Ki}_{1}}{\mathrm{Q}-\mathrm{V}_{\mathrm{t}}}-\frac{\mathrm{KQV}_{\mathrm{t}}}{\mathrm{Q}-\mathrm{V}_{\mathrm{t}}}+\mathrm{E} \cdot \exp \left(-\mathrm{D} \cdot \mathrm{V}_{\mathrm{t}}\right) \\
\mathrm{f}_{1}\left(\mathrm{~V}_{\mathrm{t}}, \mathrm{i}_{\mathrm{l}}, \mathrm{i}\right)=\mathrm{E}_{0}-\frac{\mathrm{KQi}_{\mathrm{l}}}{\mathrm{V}_{\mathrm{t}}+0.1 \mathrm{Q}}-\frac{\mathrm{KQV}}{\mathrm{Q}-\mathrm{V}_{\mathrm{t}}} \\
+\mathrm{E} \cdot \exp \left(-\mathrm{D} \cdot \mathrm{V}_{\mathrm{t}}\right) \\
\mathrm{SOC}=100\left(1-\frac{1}{\mathrm{Q}} \int_{0}^{\mathrm{t}} \mathrm{i}(\mathrm{t}) \mathrm{dt}\right)
\end{gathered}
$$

\subsection{Ultracapacitor model}

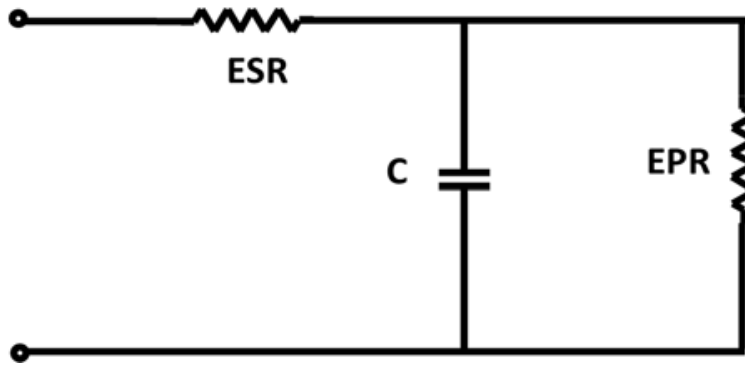

Figure 4. General representation of the UC

Different parameters of UC are

$$
\begin{aligned}
& \mathrm{V}=\frac{\mathrm{NN}_{\mathrm{s}} \mathrm{QX} 2}{\mathrm{NN}_{\mathrm{p}} \mathrm{N}^{\wedge} 2 \varepsilon \varepsilon 0 \mathrm{~A}}+ \\
& \frac{\mathrm{NN}_{\mathrm{s}} 2 \mathrm{RT}}{\mathrm{F}} \alpha \cdot r \cdot \sinh \left(\frac{\mathrm{Q}}{\mathrm{N}_{\mathrm{p}} \mathrm{N}^{2 \mathrm{~A} \sqrt{8 \mathrm{RT} \varepsilon \varepsilon \mathrm{CC}}}}\right) \\
& -i(t) \\
& =A i_{0} \exp \left(\frac{\alpha \mathrm{F}\left(\frac{\mathrm{V}}{\mathrm{N}_{\mathrm{s}}}-\frac{\mathrm{V}_{\max }}{\mathrm{N}_{\mathrm{s}}}-\Delta \mathrm{V}\right)}{\mathrm{RT}}\right) \\
& \text { SOC }=\frac{\text { Qinit }-\int_{0}^{t} i(\tau) d \tau}{\text { QT }} \times 100
\end{aligned}
$$

\section{Controllers Description}

Based on the proposed control scheme two dissimilar controllers are adopted. In which FLC is one controller used to produce the switching signals to the converter switches. And the newly constructed MFB is mend for control the 
switching signals produced by the conventional FLC corresponding to the speed on the motor.

\subsection{Fuzzy logic controller (FLC)}

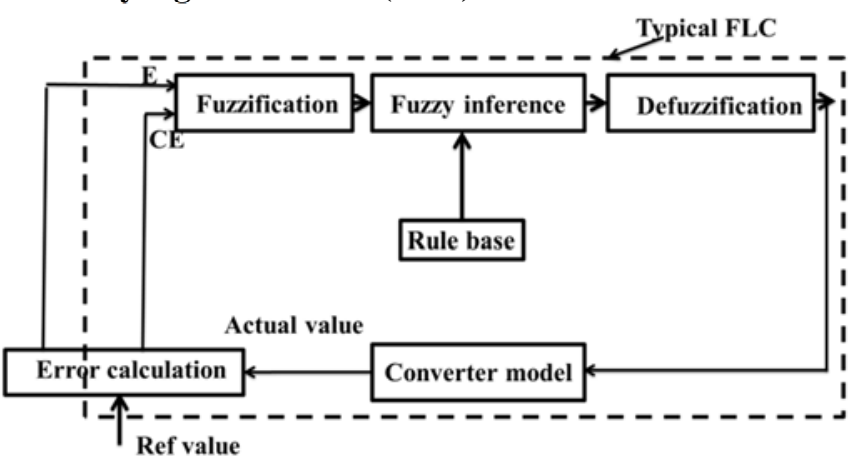

Figure 75. Generalized structure of the FLC

A typical structure of FLC has represented with figure 5. Generally separate mathematical modeling is not required for generating a rectified signal from the fuzzy logic controller. Some membership functions are selected based on the requirement of the system; generally used shapes are triangular or trapezoidal. Thereafter inference has been done by using a rule base; here rule base is used to manage the output variables. To obtain the final result from the fuzzy logic controller every rule base has been considered. Here error (E) and change in error (CE) both are considered as an input of the fuzzy logic system, that means the output of the FLC is a function of error and change in error.

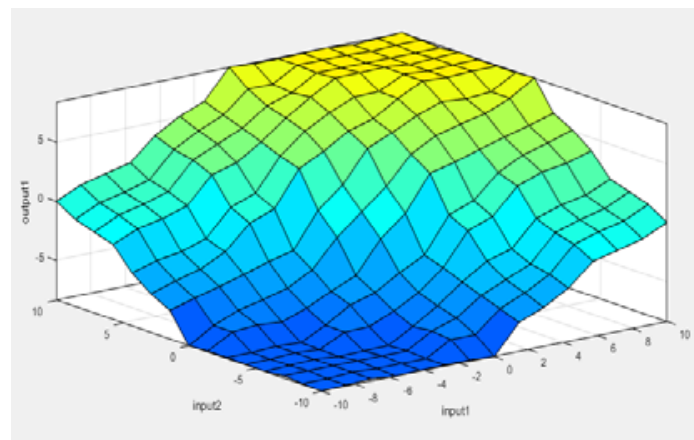

Figure 6. Surface viewer representation of the FLC

\subsection{MFB Controller}

Here MFB controller acts as a universal controller, is generating signals to four math functions, based on electric motor speed. All math functions are programmed as per the speed of the motor. Totally, MFB controller having four modes of operation. Consider the input of the MFB is speed as "N" corresponding to that MFB will produce signals to math functions U1, U2, U3, and U4 and take which as output "Y" then
(a) If $\mathrm{N} \leq 4800$ then the output becomes $\mathrm{Y}=\mathrm{U} 1$
(b) If $4600 \leq \mathrm{N} \leq 4800$ then the output becomes $\mathrm{Y}=\mathrm{U} 1 \& \mathrm{U} 2$
(c) If $4801 \leq \mathrm{N} \leq 4930$ then the output becomes $\mathrm{Y}=\mathrm{U} 3$
(d) If $\mathrm{N} \geq 4800$ then the output becomes $\mathrm{Y}=\mathrm{U} 4$

\section{Main Circuit With Different Modes}

\subsection{Mode-I operation}

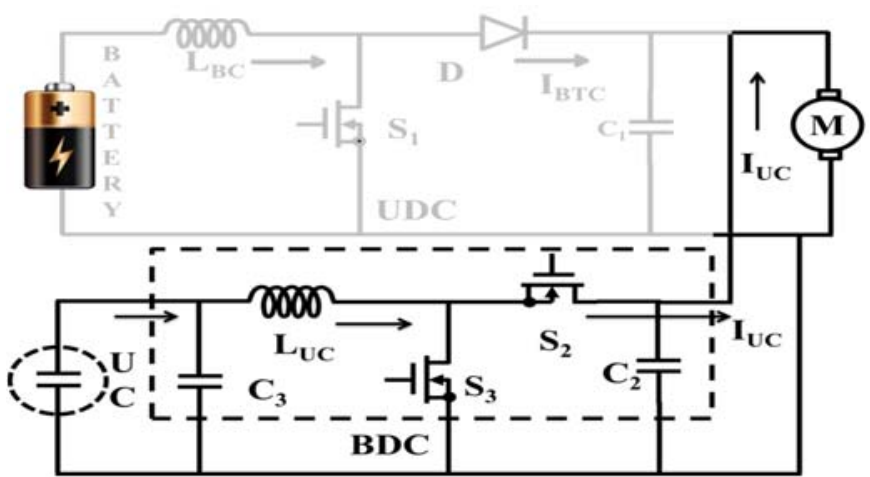

Figure 7. Main circuit with converters and sources, mode ONE

In this, switch $\mathrm{S}_{3}$ only in active state and $\mathrm{S}_{1}, \mathrm{~S}_{2}$ are in OFF state. In this case, heavy load condition on the motor. The motor requires huge current during starting and heavy load condition, in order to give the peak power necessity UC has come into the picture because UC can capable of meeting the high-power densities. Pulses have been produced to only BDC and there are no pulse signals produced to UDC. Here BDC works in boost mode to meet the peak power requirement which is related to the UC end and the power flows from UC to electric motor only.

\subsection{Mode-II operation}

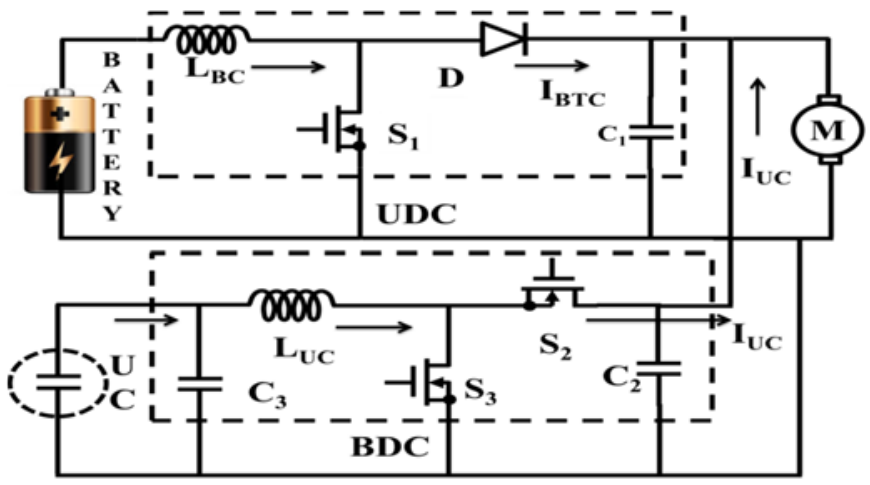

Figure 8. Main circuit with converters and sources, mode TWO

Here the slightly heavy load is applied to the motor. Battery and UC collectively meet the power required by the electric motor. The switches $S_{1}, S_{3}$ are will be in active state and $S_{2}$ is in OFF state. So, the switching signals are produced to both BDC and UDC, both will operate under boost mode. The power flows from UC to the electric motor as well as a battery to the electric motor.

\subsection{Mode-III operation}




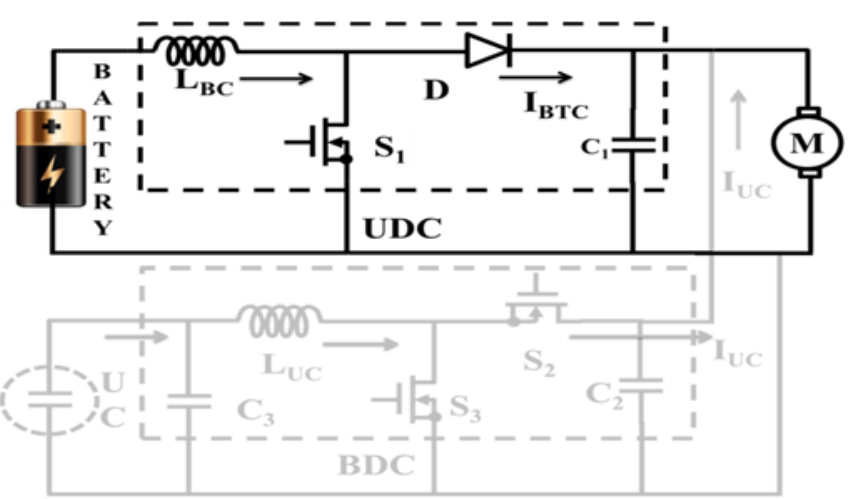

Figure 9. Main circuit with converters and sources, mode THREE

Mode three is associated with the normal load on the motor. During this state, the motor requires average power only no need for peak power requirement. Switch $S_{1}$ is in an active state and switches $S_{2}, S_{3}$ are in OFF position. So entire power required to the motor can feed by the battery only. The pulses signals generated to only UDC and there are no pulse signals produced to UDC.

\subsection{Mode-IV operation}

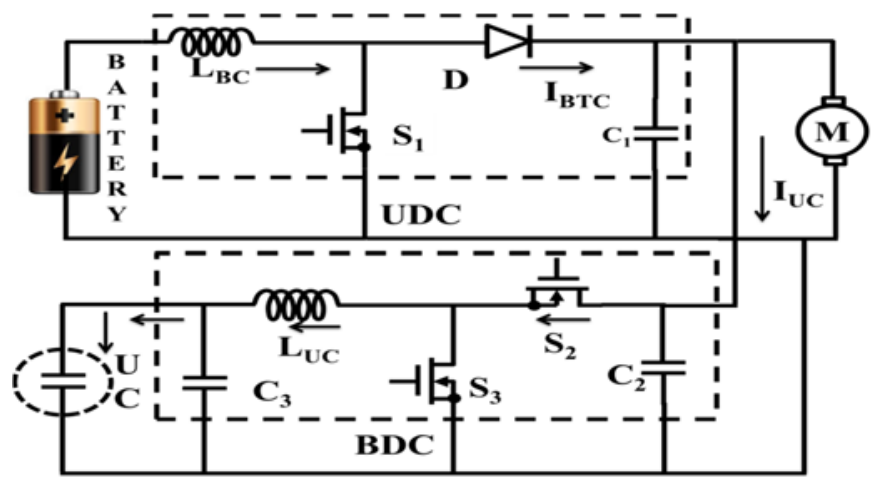

Figure 10. Main circuit with converters and sources, mode FOUR

Figure 12 represents the mode-IV operation of the circuit, during this condition switches $S_{1}, S_{2}$ are in an active state and $\mathrm{S}_{3}$ is in OFF state. The power required to the motor and, for UC charging can be supplied by the battery only. In this state, the motor is running with load free, so the motor requires normal power only. That means during mode four UC is charging through BDC (buck). The pulse signal has been produced to BDC (buck) and UDC (boost). So, the power flows from the battery to UC and electric motor.

\section{Control Strategy Approach For The Proposed Hess Topology}

The battery and UC collectively give the energy demanded by the electric motor in all conditions depending on the speed range of the motor. Here source switching can take place with the load changes on the motor. In this work, different types of loads have been taken in four modes like a heavy load, slightly greater than the rated load, rated load and no load condition. In the first mode of operation UC only produces the demanded power of the motor, during the second mode, UC and battery collectively produce the power to the motor, in third mode battery only meets the required power of the motor and in the fourth mode, the battery can give power to the motor and UC for charging. The entire circuit model operation can be done in four modes.

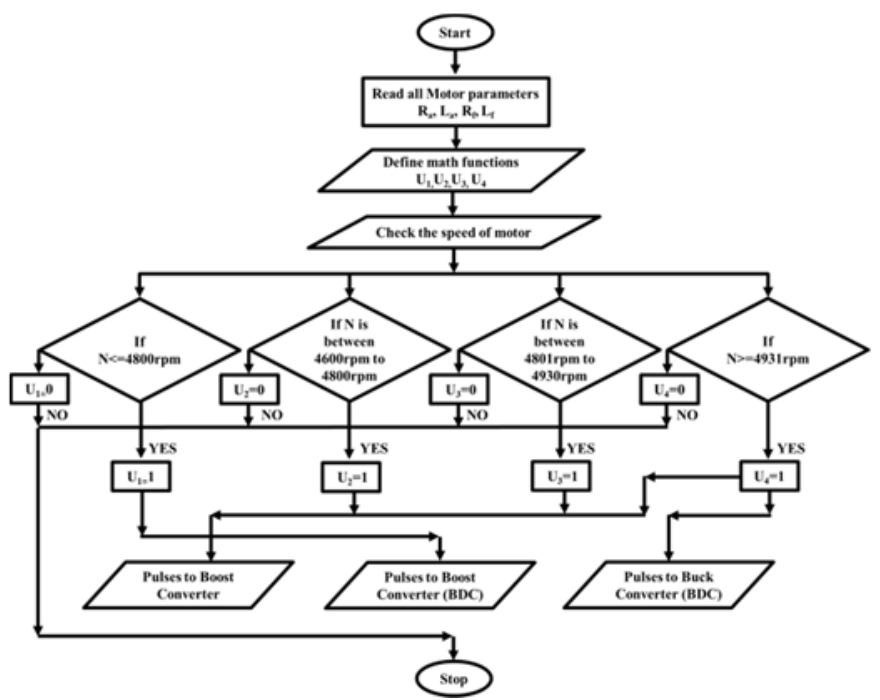

Figure 11. Production of switching signals to converters represented in the flow chart

Control strategy scheme can be implemented as follows $\mathrm{x}=$ input of MFB controller which is a variable $\mathrm{y}=$ output variable vector of MFB controller

$$
\begin{gathered}
y=f(x) \text { then } \\
f(x)=y_{0} \text { when } x \leq a \\
f(x)=y_{0}, y_{1} \text { when } b \leq x \leq c \\
f(x)=y_{2} \text { when } x \leq d \\
f(x)=y_{3} \text { when } x \geq e
\end{gathered}
$$

Here $\boldsymbol{a}, \boldsymbol{b} \boldsymbol{c}, \boldsymbol{d}, \boldsymbol{e}$ are different variable values of "which are given to MFB controller as an input. And $\boldsymbol{y}_{0} \boldsymbol{y}_{1} \boldsymbol{y}_{2}$ and $\boldsymbol{y}_{3}$ are out put vectors of the MFB controller corresponding to input values of " $\mathrm{x}$ ".

Pulse signal to switch $S_{1}$ : if $y_{1}$ or $y_{2}$ or $y_{3}$ of MFB controller present then pulse signals has been given to switch $\mathrm{S}_{1}$.

Pulse signal to switch $\mathbf{S}_{2}$ : if $\boldsymbol{y}_{3}$ of MFB controller present case then the pulse signal has been given to the switch $\mathrm{S}_{2}$.

Pulse signal to switch $S_{3}$ : if $y_{0}$ or $y_{1}$ of MFB controller present then the pulse signal can be generated to the switch $\mathrm{S}_{3}$.

\section{MATLAB/Simulation Results}

\subsection{Mode-I Results}
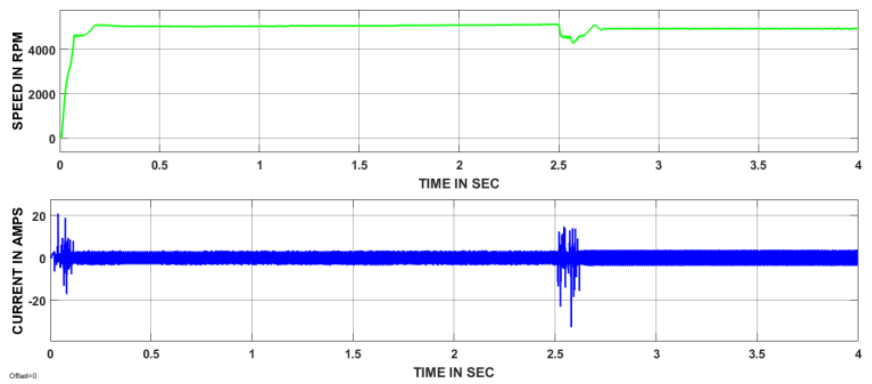

Figure 12. The output responses of the motor, mode ONE

In this case, the motor attains the stable state within $0.1 \mathrm{sec}$, starting time. After that speed, curve attains the stable state and it will continue till load applied. At $2.5 \mathrm{sec}$ heavy load is applied, which causes more disturbances in speed and current. 
Thereafter motor attains the stable state within 0.25 sec by the proposed control scheme MFB with FLC.
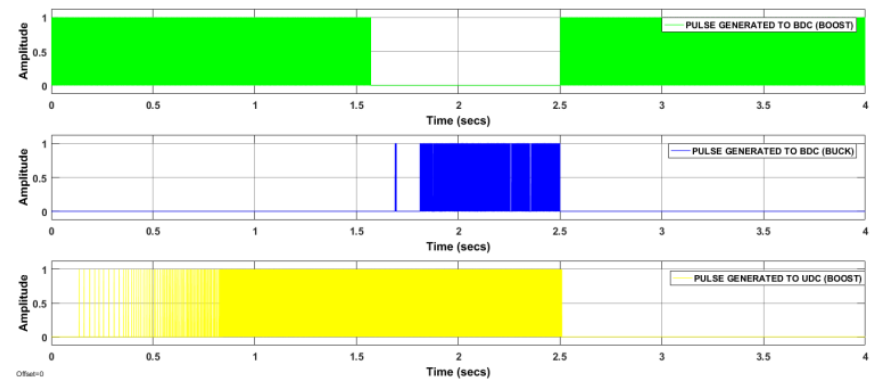

Figure 13. The controlled switching signal production by MFB with FLC, mode ONE

At the time of starting the controlled signal are produced to only BDC (boost) to provide the power demand by the load. After 0.1 sec controlled pulses are provided to UDC (boost) and BDC (buck) till the load is applied. After applying load at $2.5 \mathrm{sec}$ again the production of the switching signal is changed to only BDC (boost) and no switching signals to UDC. Thereafter motor attains stable state within $0.25 \mathrm{sec}$, due to which again the controlled signals production changes to BDC (buck) and UDC (boost), and this will continue corresponding to the vehicle dynamics by the proposed controller action.
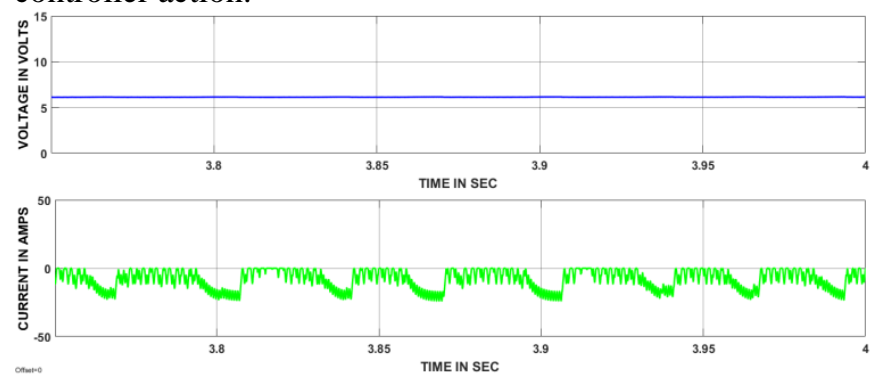

Figure 14. The input parameters of BDC, mode ONE
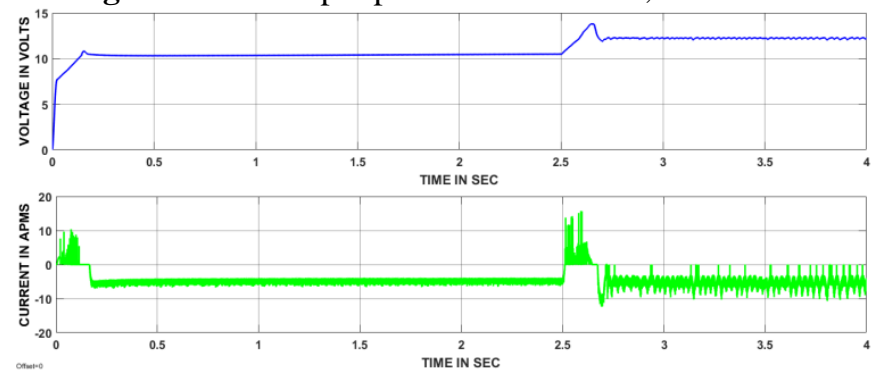

Figure 15. Outcomes Of BDC, mode ONE
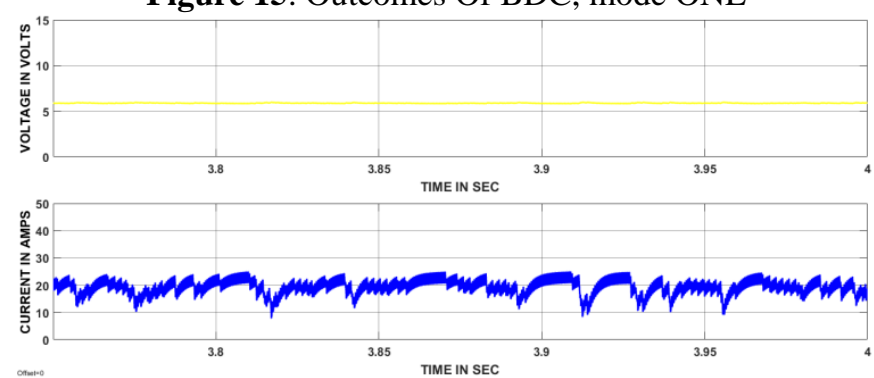

Figure 16. The input parameters of UDC, mode ONE
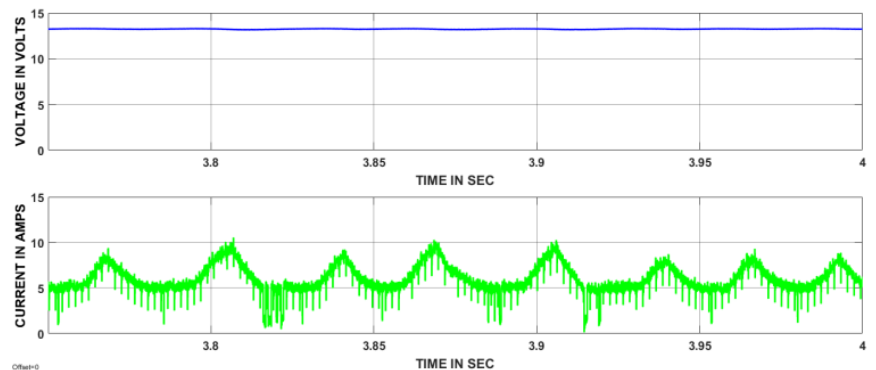

Figure 17. Outcomes Of UDC, mode ONE 7.2 Mode-II Results
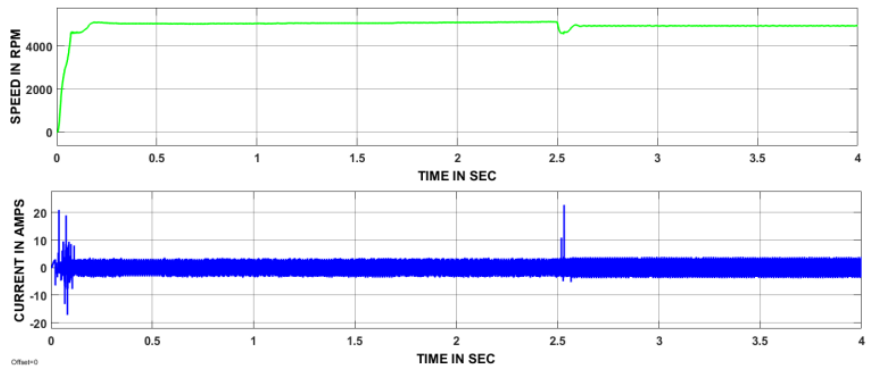

Figure 18. The output responses of the motor, mode TWO

During this mode, the motor attains the stable state within $0.1 \mathrm{sec}$, starting time. After that speed, curve attains the stable state and it will continue till load applied. At $2.5 \mathrm{sec}$ a bit more than rated load is applied, which causes disturbances in speed and current. Thereafter motor attains the stable state within $0.1 \mathrm{sec}$ by the proposed control scheme MFB with FLC.

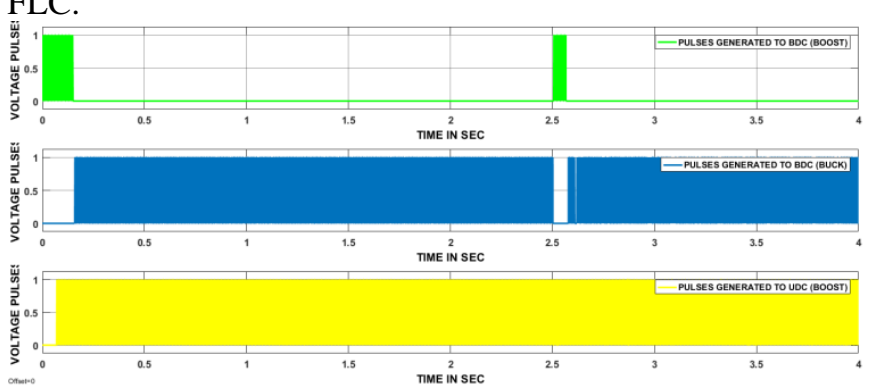

Figure 19. The controlled switching signal production by MFB with FLC, mode TWO

At the time of starting the controlled signal are produced to only BDC (boost) to provide the power demand by the load. After 0.1 sec controlled pulses are provided to UDC (boost) and BDC (buck) till the load is applied. After applying load at $2.5 \mathrm{sec}$ again the production of the switching signal is changed to only BDC (boost) and no switching signals to UDC. Thereafter motor attains stable state within $0.1 \mathrm{sec}$, due to which again the controlled signals production changes to BDC (buck) and UDC (boost), and this will continue corresponding to the vehicle dynamics by the proposed controller action.

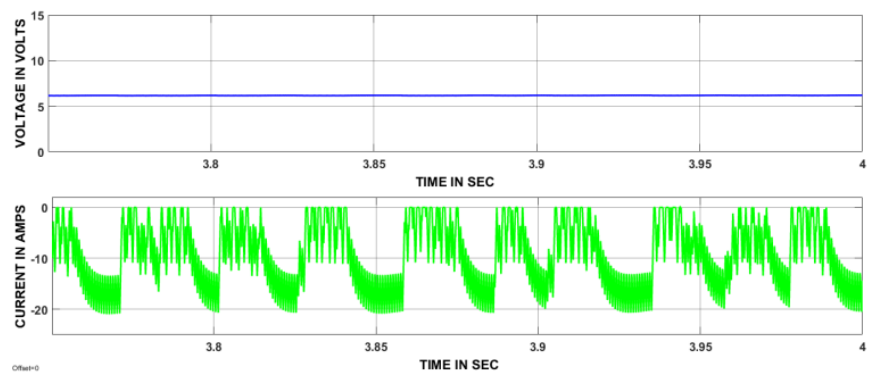

Figure 20. The input parameters of BDC, mode TWO 

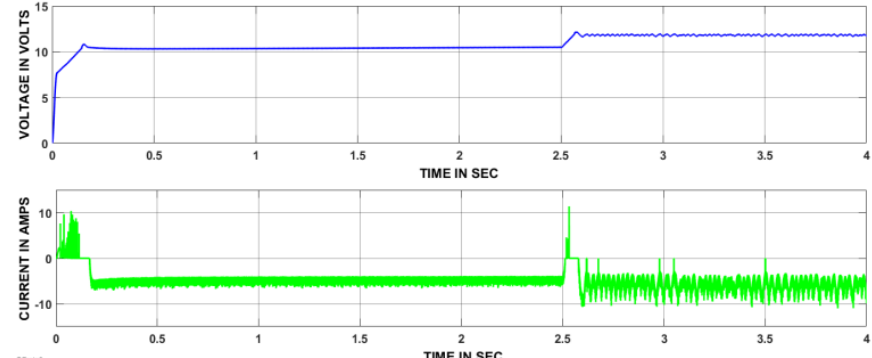

Figure 21. Outcomes Of BDC, mode TWO
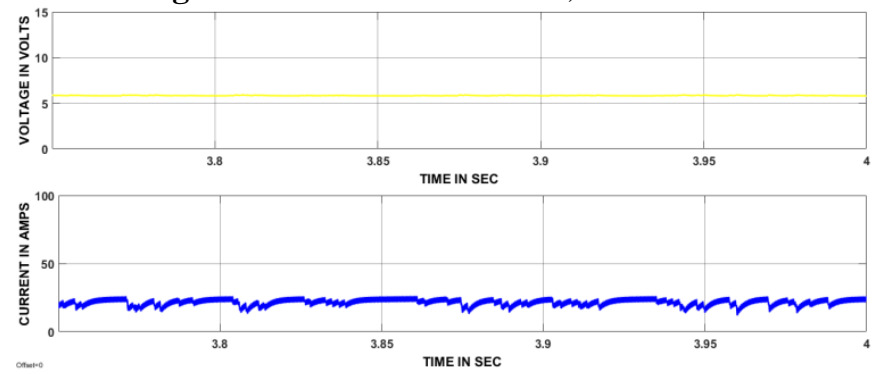

Figure 22. The input parameters of UDC, mode TWO
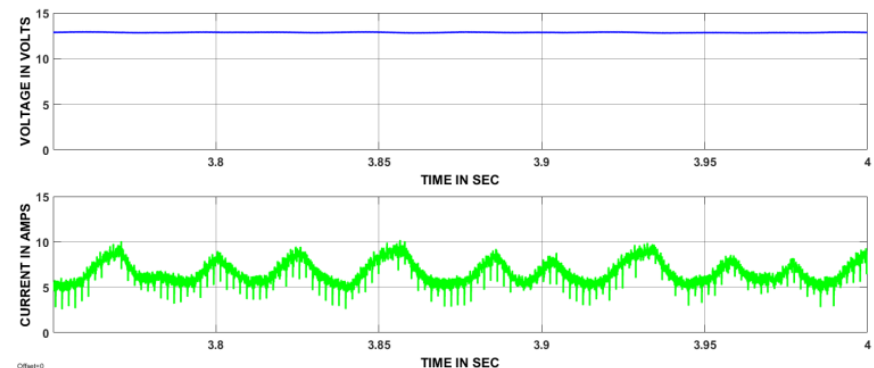

Figure 23. Outcomes Of UDC, mode TWO

\subsection{Mode-III Results}
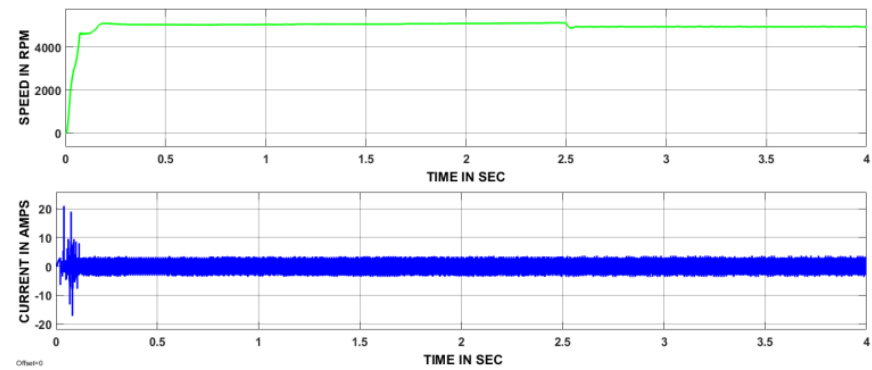

Figure 24. The output responses of the motor, mode THREE

In this case, the motor attains the stable state within $0.1 \mathrm{sec}$, starting time. After that speed, curve attains the stable state and it will continue till load applied. At 2.5 sec rated load is applied, which causes normal disturbances in speed and current. Thereafter motor attains the stable state within 0.04 sec by the proposed control scheme MFB with FLC.
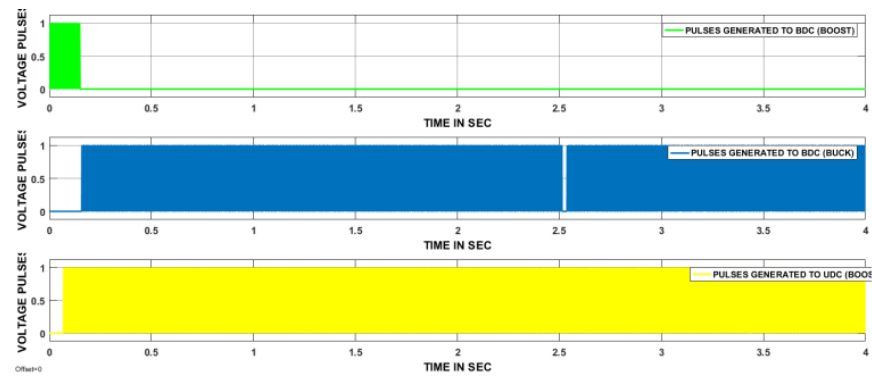

Figure 25. The controlled switching signal production by MFB with FLC, mode THREE

At the time of starting the controlled signal are produced to only BDC (boost) to provide the power demand by the load. After 0.1 sec controlled pulses are provided to UDC (boost) and BDC (buck) till the load is applied. After applying load at $2.5 \mathrm{sec}$ again the production of the switching signal is changed to only BDC (boost) and no switching signals to UDC. Thereafter motor attains stable state within $0.05 \mathrm{sec}$, due to which again the controlled signals production changes to BDC (buck) and UDC (boost), and this will continue corresponding to the vehicle dynamics by the proposed controller action.
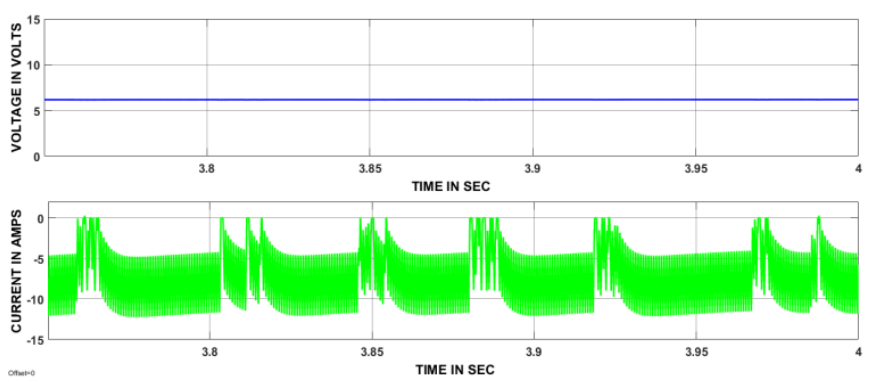

Figure 26. The input parameters of BDC, mode THREE
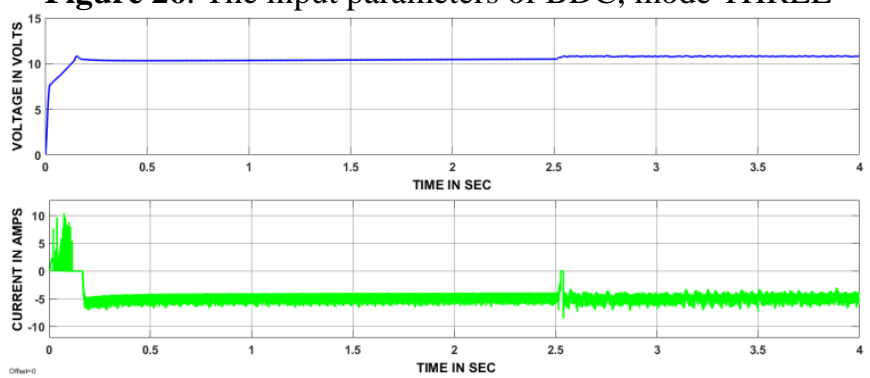

Figure 27. Outcomes Of BDC, mode THREE

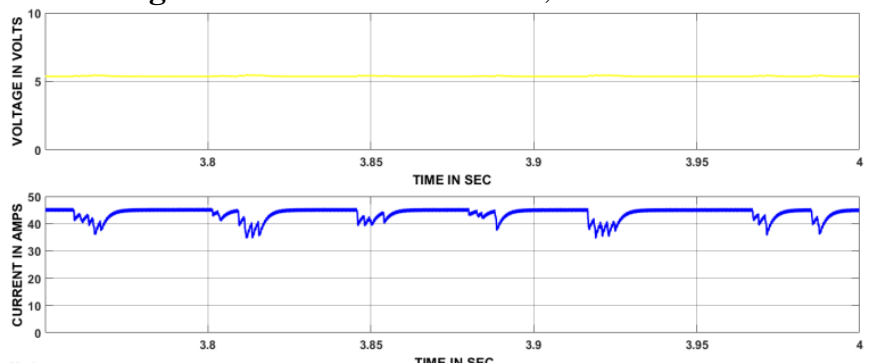

Figure 28. The input parameters of UDC, mode THREE
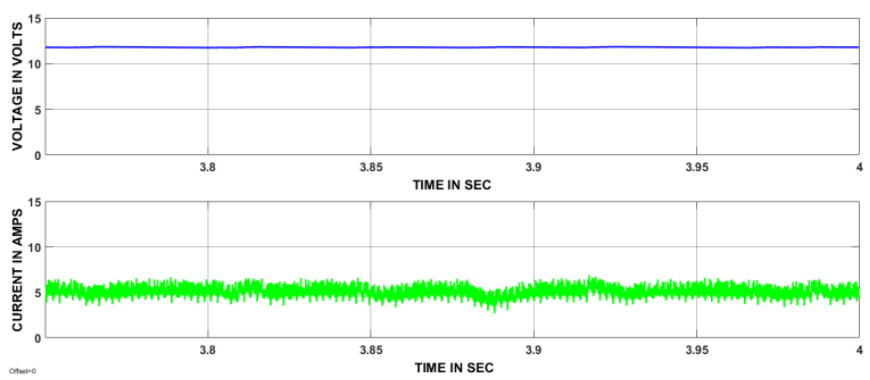

Figure 29. Outcomes Of UDC, mode THREE 7.4 Mode-IV Results 

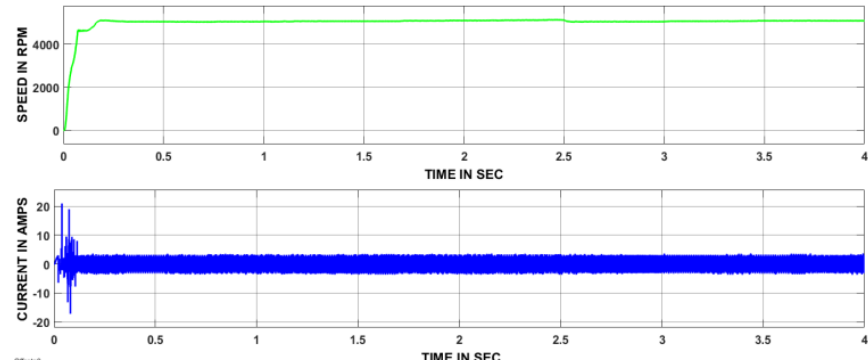

Figure 30. The output responses of the motor, mode FOUR

During this mode, the motor attains the stable state within $0.1 \mathrm{sec}$, starting time. After that speed, curve attains the stable state and it will continue till load applied. Under this mode, the motor runs with no load.

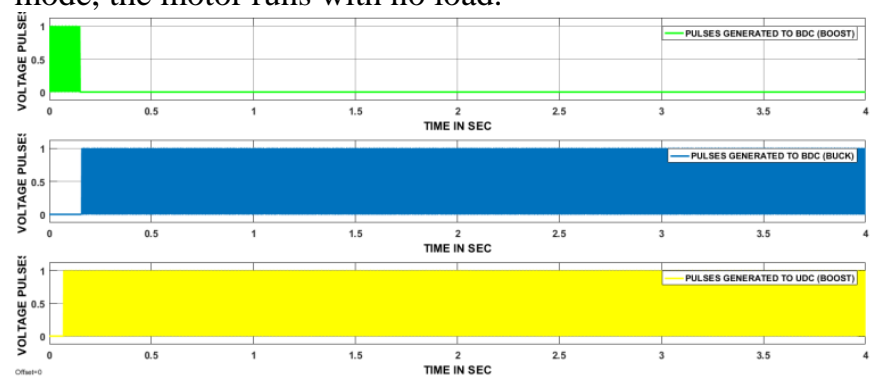

Figure 31. The controlled switching signal production by MFB with FLC, mode FOUR

At the time of starting the controlled signal are produced to only BDC (boost) to provide the power demand by the load. After 0.1 sec controlled pulses are provided to UDC (boost) and BDC (buck) till the load is applied. In this case, motor runs with free load.
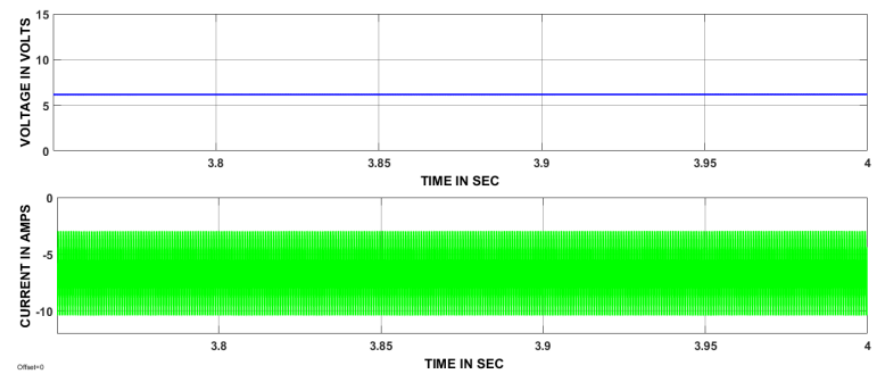

Figure 32. The input parameters of BDC, mode FOUR
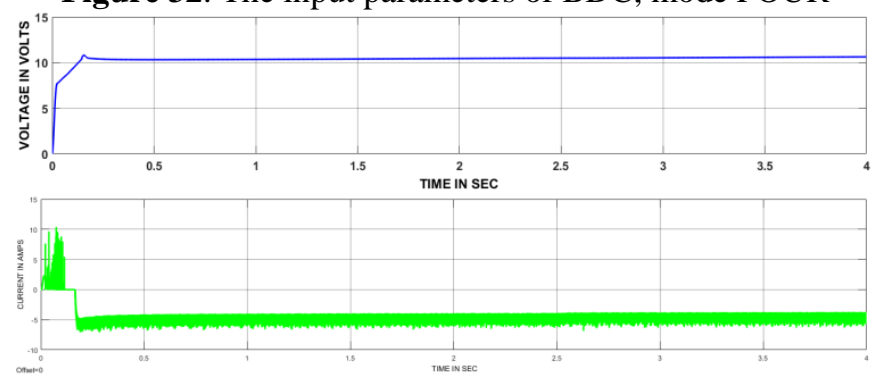

Figure 33. Outcomes Of BDC, mode FOUR
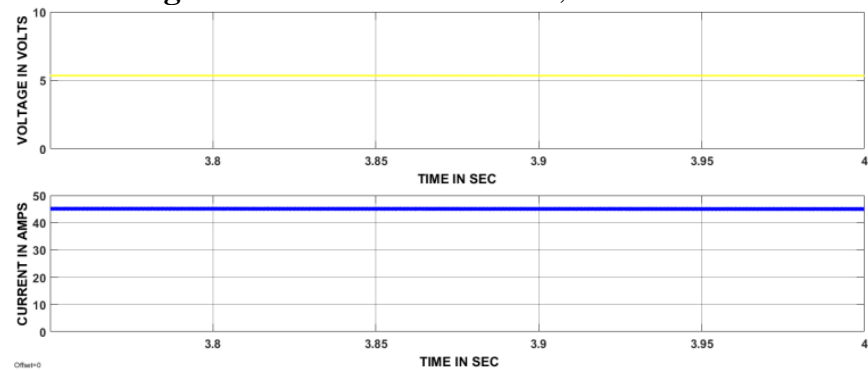

Figure 34. The input parameters of UDC, mode FOUR
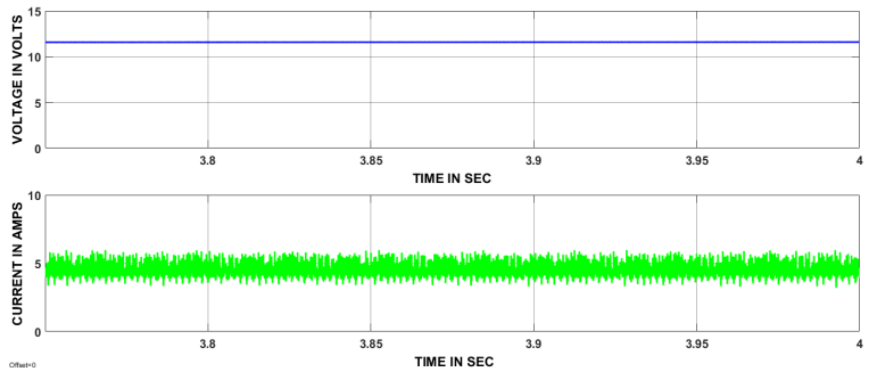

Figure 35. Outcomes Of UDC, mode FOUR

\subsection{Continuous load applied to the motor}
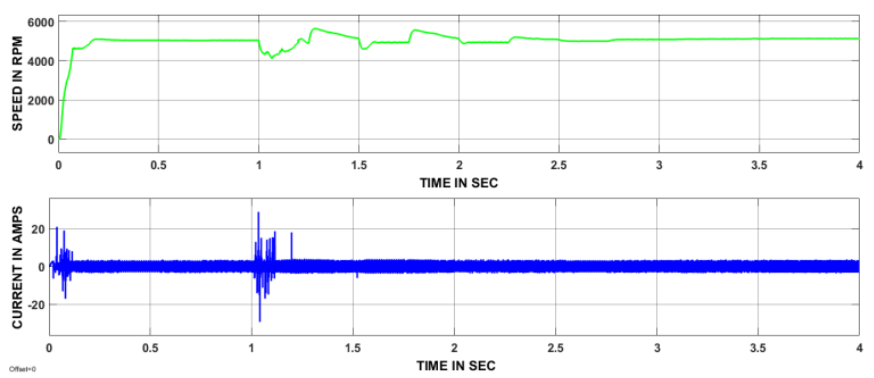

Figure 36. Speed and Current responses during a continuous load condition

From Figure 36 it is clear that the load on the motor is present at different intervals with different values. From 1 to $1.5 \mathrm{sec}$ heavy load has been applied to the motor, between 2 to $2.5 \mathrm{sec}$ medium load and between 3 to $3.5 \mathrm{sec}$ normal loads are applied to the motor. According to the load variations, the speed of the motor is also changing. Subsequently, the armature current of the motor is also changing with load variations. During the loaded conditions speed of the motor is reducing and increasing the armature current value and remaining time the electric motor is maintaining the rated speed and rated current values.

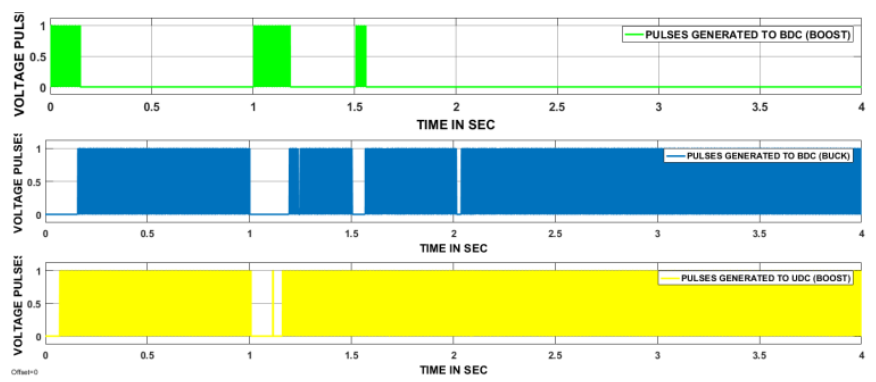

Figure 37. The controlled switching signal production by MFB with FLC, Continuous mode

During heavy load and starting of the electric motor, UC only provides power to the motor so corresponding to this mode BDC operates in Boost mode. Slightly more than normal load condition battery and UC collectively provide power to the motor, BDC works in Boost mode and UDC. During rated load condition battery only provide power to the motor, only UDC work in Boost mode. During no-load condition, the battery give power to motor and UC. 

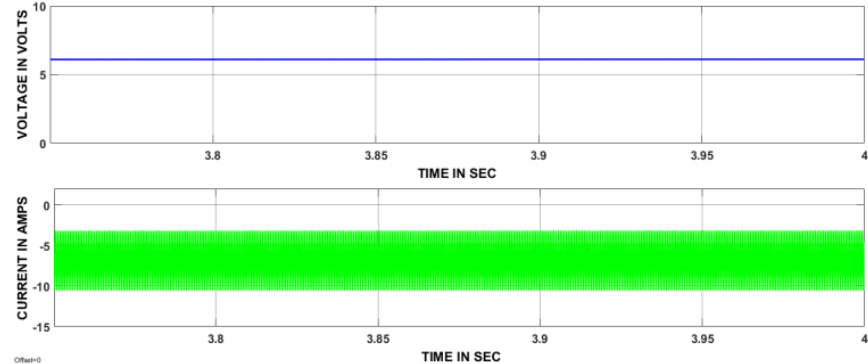

Figure 38. The input parameters of BDC, continuous mode

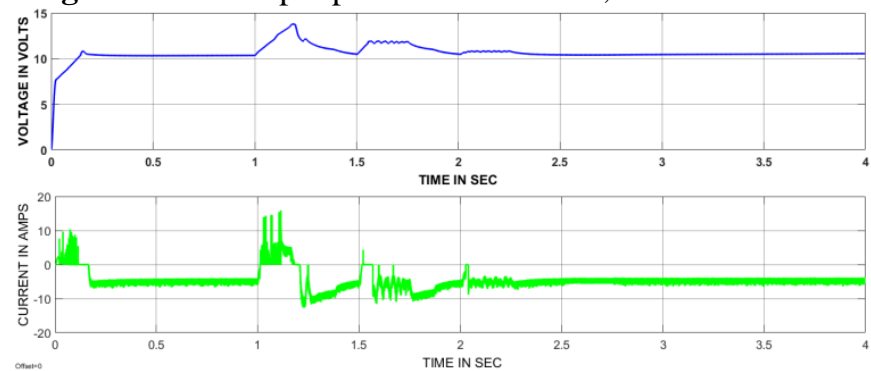

Figure 39. Outcomes Of BDC, Continuous mode
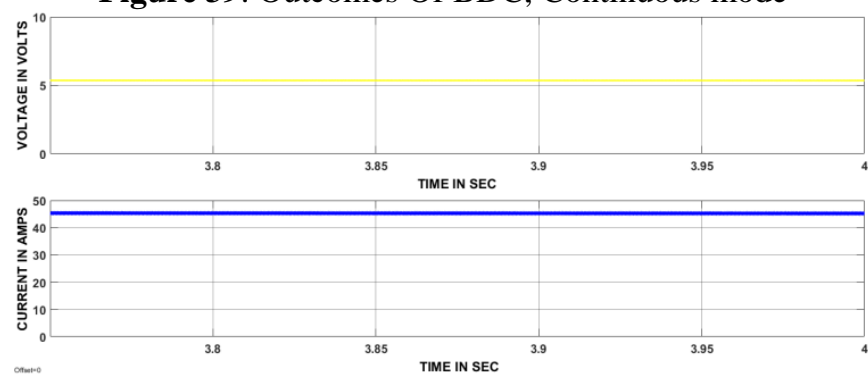

Figure 40. The input parameters of UDC, continuous mode
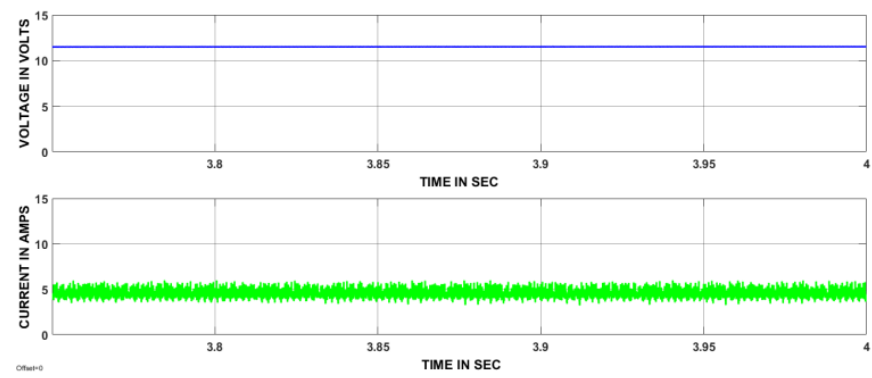

Figure 41. Outcomes Of UDC, Continuous mode

Table 1. state of operation of MFB corresponding to the speed

\begin{tabular}{ccc}
\hline S.No & Speed value & $\begin{array}{c}\text { ON state math } \\
\text { function }\end{array}$ \\
\hline 1 & $\mathrm{~N} \leq 4800 \mathrm{rpm}$ & $\mathrm{U}_{1}=1$ \\
\hline 2 & $4600 \mathrm{rpm} \leq_{\mathrm{N}} \leq_{4800}$ & $\mathrm{U}_{1}=1 \& \mathrm{U}_{2}=1$ \\
\hline 3 & $4801 \mathrm{rpm} \leq \mathrm{N} \leq 4930$ & $\mathrm{U}_{3}=1$ \\
\hline 4 & $\mathrm{~N} \geq_{4931 \mathrm{rpm}}$ & $\mathrm{U}_{4}=1$ \\
\hline
\end{tabular}

\section{Conclusions}

MFB controller is integrated with fuzzy logic and implemented as a hybrid controller to an electric vehicle for a smooth transition between battery and UC. MFB controller has always regulated the switching signals developed by the fuzzy logic controller corresponding to the speed of the motor. During mode-I, pulses are produced to switch S3 only which means that the power is power flows from UC to load. In mode-II operation-controlled pulses are produced to switches $S_{1}$ and $S_{3}$ which indicates that energy required by the electric vehicle is supplied by both battery and UC. During mode-III operation switch $\mathrm{S}_{1}$ only in $\mathrm{ON}$ position, which indicates that battery provides essential power to the electric vehicle. In the fourth mode of operation switches, $S_{1}$ and $S_{2}$ are in an active state, and the power is transferring to UC as well as an electric motor from the battery. Finally, in four modes the designed MFB controller with fuzzy logic worked satisfactorily and switched the battery and UC according to the electric motor power requirement.

\section{References}

[1] Shengzhe, Z., Kai, W., \& Wen, X. (2017, October). Fuzzy logic-based control strategy for a battery/supercapacitor hybrid energy storage system in electric vehicles. In Chinese Automation Congress (CAC), 2017 (pp. 5598-5601). IEEE.

[2] Elwarfalli, H., Muntaser, A., Kumar, J., \& Subramanyam, G. (2016, July). Design and implementation of PI controller for the hybrid energy system. In Aerospace and Electronics Conference (NAECON) and Ohio Innovation Summit (OIS), 2016 IEEE National (pp. 170-172). IEEE.

[3] Allègre, A. L., Bouscayrol, A., \& Trigui, R. (2013). Flexible real-time control of a hybrid energy storage system for electric vehicles. IET Electrical Systems in Transportation, 3(3), 79-85.

[4] Yanzi, W., \& Wang, W. (2014, August). Energy management strategy based on fuzzy logic for a new hybrid battery-ultracapacitor energy storage system. In Transportation Electrification Asia-Pacific (ITEC AsiaPacific), 2014 IEEE Conference and Expo (pp. 1-5). IEEE.

[5] Michalczuk, M., Ufnalski, B., \& Grzesiak, L. (2013, March). Fuzzy logic control of a hybrid batteryultracapacitor energy storage for an urban electric vehicle. In Ecological Vehicles and Renewable Energies (EVER), 2013 8th International Conference and Exhibition on (pp. 1-7). IEEE.

[6] Dusmez, S., \& Khaligh, A. (2014). A supervisory power-splitting approach for a new ultracapacitorbattery vehicle deploying two propulsion machines. IEEE Transactions on Industrial Informatics, 10(3), 1960-1971.

[7] Shen, J., \& Khaligh, A. (2015). A supervisory energy management control strategy in a battery/ultracapacitor hybrid energy storage system. IEEE Transactions on Transportation Electrification, 1(3), 223-231.

[8] Yin, H., Zhou, W., Li, M., Ma, C., \& Zhao, C. (2016). An adaptive fuzzy logic-based energy management strategy on battery/ultracapacitor hybrid electric vehicles. IEEE Transactions on Transportation Electrification, 2(3), 300-311.

[9] Xiang, C., Wang, Y., Hu, S., \& Wang, W. (2014). A new topology and control strategy for a hybrid batteryultracapacitor energy storage system. Energies, 7(5), 2874-2896. 
[10] Wu, D., Todd, R., \& Forsyth, A. J. (2015). Adaptive rate-limit control for energy storage systems. IEEE Transactions on Industrial Electronics, 62(7), 42314240.

[11] Tani, A., Camara, M. B., Dakyo, B., \& Azzouz, Y. (2013). DC/DC and DC/AC converters control for hybrid electric vehicles energy management- ultracapacitors and fuel cell. IEEE Transactions on Industrial Informatics, 9(2), 686-696.

[12] Averbukh, M., Lineykin, S., \& Kuperman, A. (2015). Portable ultracapacitor-based power source for emergency starting of internal combustion engines. IEEE Transactions on Power Electronics, 30(8), 42834290. 